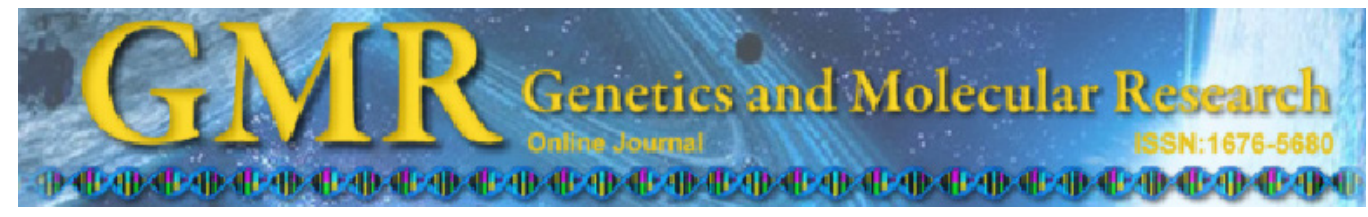

Short Communication

\title{
Microsatellite markers derived from Japanese scallop (Mizuhopecten yessoensis) expressed sequence tags
}

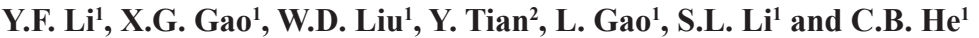 \\ ${ }^{1}$ Key Laboratory of Marine Fishery Molecular Biology, \\ Liaoning Ocean and Fisheries Science Research Institute, Dalian, China \\ ${ }^{2}$ College of Fisheries and Life Science, Dalian Ocean University, Dalian, China \\ Corresponding author: C.B. He \\ E-mail: hechongbo@hotmail.com
}

Genet. Mol. Res. 13 (1): 1989-1992 (2014)

Received December 5, 2012

Accepted April 18, 2013

Published March 24, 2014

DOI http://dx.doi.org/10.4238/2014.March.24.3

\begin{abstract}
Japanese scallop (Mizuhopecten yessoensis) is a coldwater shellfish, and a species of economic importance in China. In this study, we developed and evaluated simple sequence repeat (SSR) markers from the expressed sequence tags (ESTs) of M. yessoensis. The characteristics of 12 EST-SSR loci were investigated in 30 individual scallops, and the result revealed that the number of alleles per locus ranged from 2-4, with an observed heterozygosity ranging from $0.0333-$ 0.7692 , and an expected heterozygosity ranging from $0.0333-0.6312$. Only two loci were found to depart significantly from the HardyWeinberg equilibrium $(\mathrm{P}<0.05)$. The result of our study suggested that these markers could be considered as potential markers for studying the population structure of M. yessoensis and its intraspecific variation.
\end{abstract}

Key words: Mizuhopecten yessoensis; Expressed sequence tags; Japanese scallop; Molecular marker; Simple sequence repeats 
The Japanese scallop Mizuhopecten yessoensis is a cold-water shellfish, and its original habitat is distributed across various regions of the world, including the northern part of Japan, the far eastern part of Russia, and the eastern part of the Korean Peninsula. Due to its large body mass, good taste, and nutritional value, $M$. yessoensis has become a high-value food. In 1983, M. yessoensis was introduced to China from Japan, and since then much research effort has focused on the development of better techniques for cultivating this species of scallop. However, despite increased cultivation, recent declines in quality and yield have prompted many studies on the genetic diversity of M. yessoensis (Wang et al., 2009; Li et al., 2010; Liu et al., 2010) to address concerns regarding its protection and genetic diversity in the hope of preventing a decline in its population.

Few studies have used DNA-based methods to study the population structure of $M$. yessoensis (Sato et al., 2005; Wang et al., 2009). Microsatellite DNAs, also known as simple sequence repeats (SSRs), are short (1-6 bp in length) tandem-repeat sequences that are widely dispersed in eukaryotic genomes. SSRs have been extensively used to study the genetic diversity and population structure of many species because of their high level of polymorphisms, co-dominant inheritance, and distribution throughout the genome (Xu et al., 2010; Yang et al., 2011; Zheng et al., 2012). Expressed sequence tags (ESTs) represent part of the transcribed genome of an organism and are an important resource for identifying microsatellites (Gao et al., 2010). In this study, we identified the microsatellites from M. yessoensis ESTs and analyzed the polymorphisms present in the EST-SSRs.

The SSRs of M. yessoensis genome were obtained by screening 3009 M. yessoensis ESTs obtained in our laboratory and $4711 \mathrm{M}$. yessoensis ESTs obtained from GenBank (at http://www.ncbi.nlm.nih.gov/Genbank/). The SSR sequences were identified using the software MISA (http://pgrc.ipk-gatersleben.de/misa/) and 58 of these EST-SSRs were randomly selected for polymerase chain reaction (PCR) using M. yessoensis genomic DNA as template. PCR primers were designed using the Primer 5.0 software with default parameters.

M. yessoensis genomic DNA was extracted from 30 different individuals obtained from a cultured population in Dalian, China. The DNA was extracted by a conventional phenol/chloroform extraction method. Each PCR reaction was conducted in a $25-\mu \mathrm{L}$ volume containing $50 \mathrm{ng}$ genomic DNA, 1X PCR buffer, $1.5 \mathrm{mM} \mathrm{MgCl}, 0.2 \mathrm{mM}$ dNTPs, $200 \mathrm{nM}$ of each primer, and $1 \mathrm{U}$ Taq polymerase (Takara). The PCR condition consisted of an initial denaturation at $94^{\circ} \mathrm{C}$ for $5 \mathrm{~min}$, followed by 35 cycles of denaturation at $94^{\circ} \mathrm{C}$ for $30 \mathrm{~s}$, annealing at one of the temperatures shown in Table 1 for $30 \mathrm{~s}$, and extension at $72^{\circ} \mathrm{C}$ for $30 \mathrm{~s}$, with a final extension step at $72^{\circ} \mathrm{C}$ for $10 \mathrm{~min}$. The amplified-PCR products were separated in $10 \%$ nondenaturing polyacrylamide gel at $280 \mathrm{~V}$ for $1-2 \mathrm{~h}$. After electrophoresis the gel was stained with ethidium bromide and visualized under ultraviolet light. The genetic diversity indices, including the observed and expected heterozygosities and tests for departure from the HardyWeinberg equilibrium (HWE) were performed using POPGENE32 version 1.32.

Of the 58 primer pairs tested, 22 did not generate the expected PCR products, whereas 24 produced monomorphic bands, while 12 produced polymorphic bands. In these 12 polymorphic bands, the number of alleles per locus ranged from 2-4 (Table 1). Overall, the extent of polymorphism associated with the EST-SSRs in M. yessoensis genome appeared to be low, indicating M. yessoensis may have low genetic diversity. This may also reflect the presence of germplasm degeneration. The observed heterozygosity ranged from $0.0333-0.7692$, whereas the expected heterozygosity ranged from $0.0333-0.6312$. Departure from the Hardy-Weinberg equilibrium $(\mathrm{P}<0.05)$ was only observed for the loci P1 and P10 (Table 1). 


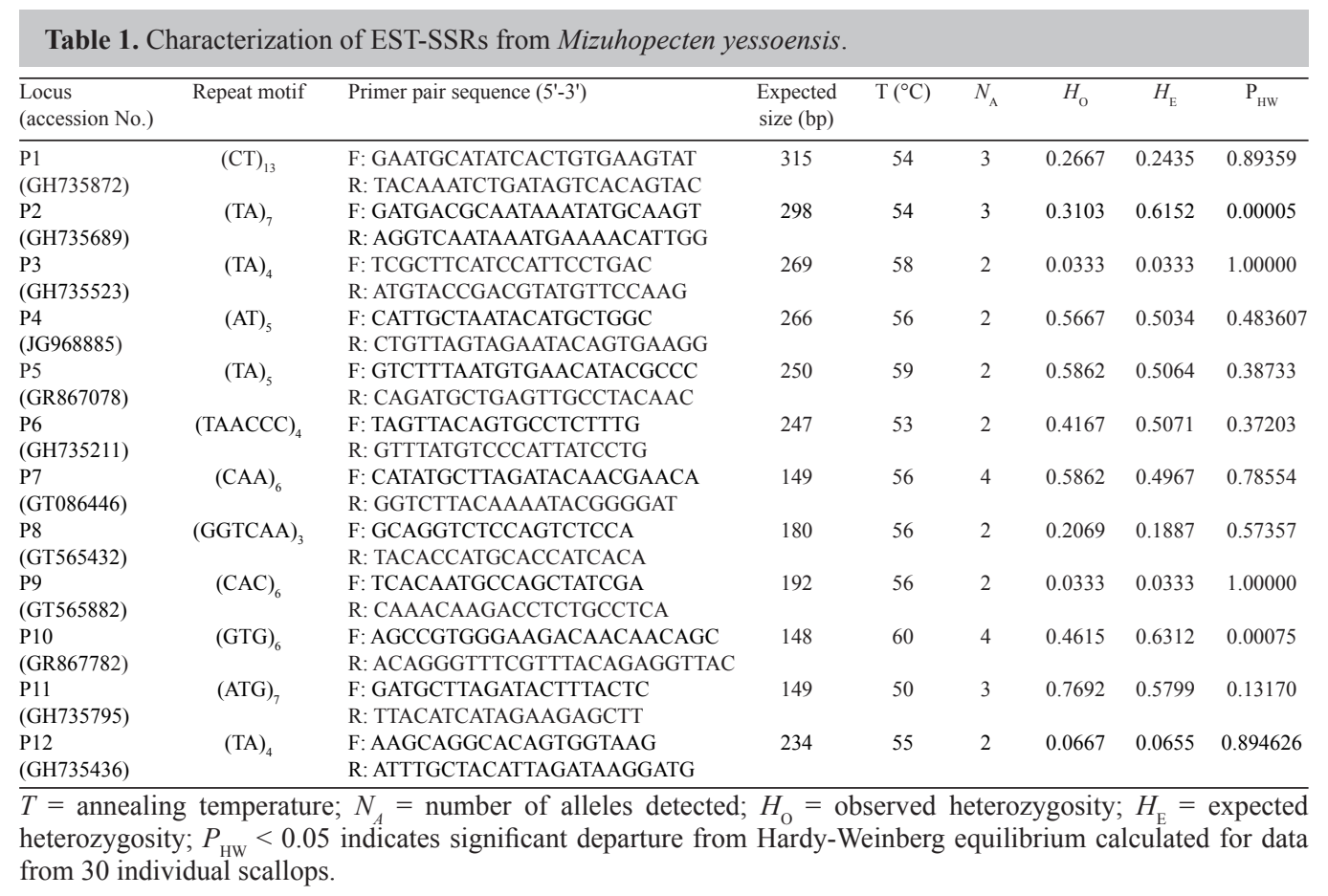

The polymorphic microsatellites derived from M. yessoensis identified in this study will be useful for analyzing the genetic structures of its population. It will also facilitate future studies to assess the genetic diversity within this commercially important scallop.

\section{ACKNOWLEDGMENTS}

Research supported by grants from the National Nature Science Foundation of China (\#31302173, \#31302178, and \#30972246), the Program of Liaoning Province Science and Technology Commission (\#2008203002), and the Liaoning Scientific Research Program of Ocean and Fisheries Department (\#200801).

\section{REFERENCES}

Gao XG, Li HJ, Li YF, Sui LJ, et al. (2010). Sixteen polymorphic simple sequence repeat markers from expressed sequence tags of the Chinese Mitten Crab Eriocheir sinensis. Int. J. Mol. Sci. 11: 3035-3038.

Li YF, Liu WD, Gao XG and Li WD (2010). Construction of cDNA libraries from mantle and kidney of Japanese scallop (Mizuhopecten yessoensis) and ESTs analysis. J. Fish. Sci. China 3: 578-585.

Liu WD, Li HJ, Bao XB, Gao XG, et al. (2010). Genetic differentiation between natural and hatchery stocks of Japanese scallop (Mizuhopecten yessoensis) as revealed by AFLP analysis. Int. J. Mol. Sci. 11: 3933-3941.

Sato M, Kawamata K, Zaslavskaya N, Nakamura A, et al. (2005). Development of microsatellite markers for Japanese scallop (Mizuhopecten yessoensis) and their application to a population genetic study. Mar. Biotechnol. 7: 713-728.

Wang XL, Song B, Qiu XM and Meng XY (2009). Development of EST-SSRs in scallop (Patinopecten yessoensis) from sequence database. Cons. Genet. 10: 1129-1131. 
Xu TJ, Sun YN, Yuan YT, Liao Z, et al. (2010). Isolation and characterization of polymorphic microsatellite loci in the hard-shelled mussel, Mytilus coruscus (Mytilidae). Genet. Mol. Res. 9: 1388-1391.

Yang S, Wang L, Zhang Y, Liu XC, et al. (2011). Development and characterization of 32 microsatellite loci in the giant grouper Epinephelus lanceolatus (Serranidae). Genet. Mol. Res. 10: 4006-4011.

Zheng XD, Zuo ZR, Su XJ and Li Q (2012). Isolation and characterization of 24 polymorphic microsatellite loci in Sepioteuthis lessoniana. Genet. Mol. Res. 11: 3961-3965. 